\title{
THE ENTROPY OF DISCRETE-TIME FRACTIONAL GAUSSIAN NOISE AND ITS APPLICATION TO THE ELECTROMYOGRAM OF EXTERNAL URETHRAL SPHINCTER SIGNALS
}

\author{
YeN-Ching Chang AND SHYANG CHANG \\ Department of Electrical Engineering, National Tsing Hua University \\ Hsinchu, Taiwan
}

\section{ABSTRACT}

In this work, we will study the Hurst parameter embedded in the discrete-time fraction Gaussian noise (DFGN) and derive the entropy with this parameter. It will be applied to the electromyogram $(E M G)$ of external urethral sphincter (EUS). These signals come from intact rats and the injured ones from spinal cord injury (SCI). Analysis indicates that we can discriminate between intact and SCI rats from this information.

Biomed Eng Appl Basis Comm, 2001 (December); 13: 256-261.

Key words: fraction Brownian motion, discrete-time fractional Brownian motion, discrete-time Gaussian noise, Hurst parameter, external urethral sphincter, electromyogram.

\section{INTRODUCTION}

In recent years, fractional Brownian motion (FBM) model has been widely applied to a large number of natural shapes and phenomena that used to be represented by the traditional autoregressive moving average (ARMA) models. For digital applications, we must sample the FBM. The sampled FBM will be termed as discrete-time fractional Brownian motion (DFBM). However, the nonstationality of DFBM is not suitable for application. Meanwhile, the increment of DFBM, referred to as discrete-time fraction Gaussian noise (DFGN), is stationary and the probability density function (PDF) is jointly Gaussian. In many applications, the DFGN is used to estimate the parameter Hurst exponent $H$ in order to discriminate among the data derived [1] [2]. Hurst exponent is confined in the re-

Received: Oct. 17, 2001; accepted: Nov. 12, 2001 Correspondence: Shyang Chang, Professor Department of Electrical Engineering National Tsing Hua Univerrsity, Hsinchu, Taiwan E-mail: shyang@ce.nthu.edu.tw gion $(0,1)$. Furthermore, this parameter possesses the relation $H=2-D$ with $D$, the fractal dimension [3]. Many estimators [4]-[12] have been presented to estimate this parameter $D$, i.e., the fractal dimension, which can be used to describe how rough a curve is. A curve is more smooth if $D$ is closer to 1 in dimension. A curve is more rough if $D$ is closer to 2 in dimension.

Since the PDF of DFGN is jointly Gaussian, we can easily derive the entropy with Hurst parameter. This entropy is also suitable for the description of many real signals. In this work, we will use this property on DFGN to discriminate between the EMGs of the intact rats and the $\mathrm{SCI}$ ones.

This paper is organized as follows. Some terminologies and preliminaries are introduced in Section 2. In Section 3, we derive the entropy property. In Section 4, we apply the property to EMG signals. Finally, we conclude this paper in Section 5 .

\section{METHOO}

\subsection{Terminologies and Preliminaries}


Some definitions and their properties will be introduced in this paragraph for reference. At this time, we will use $\{x(t), t \in R\}$ to designate a continuoustime process, $\{x[n] n \in Z\}$ for a discrete-time process for convenience.

The FBM is a generalization of the ordinary Brownian motion. According to the Mandelbrot and Van Ness [13], FBM can be expressed as follows:

$$
\begin{aligned}
& B_{H}(0, \omega)=b_{0} \\
& B_{H}(t, \omega)-B_{H}(0, \omega) \\
& =\frac{1}{\Gamma(H+1 / 2)}\left\{f_{-}^{0}\left[(t-s)^{\mu \cdot v^{2}}-(-s)^{H \cdot v_{2}}\right] d B(s, \omega)\right. \\
& \left.\quad+\int_{0}(t-s)^{H-v_{2}} d B(s, \omega)\right\}
\end{aligned}
$$

where $H$ is a Hurst exponent between 0 and 1 , and $B(s, \omega)$ is the ordinary Brownian motion. The integration is operated in the pointwise sense as well as the mean square sense by using integration by parts. It is obvious that, FBM with $H=0.5$ coincides with ordinary Brownian motion. The definition (1) of $B_{H}$ can be made more symmetric, including a convergent difference of two divergent integrals, as follows:

$$
\begin{aligned}
& B_{H}\left(t_{2}, \omega\right)-B_{H}\left(t_{1}, \omega\right) \\
= & \frac{1}{\Gamma(H+1 / 2)}\left\{\int_{-\infty}^{z_{2}}\left(t_{2}-s\right)^{H-v_{2}} d B(s, \omega)\right. \\
& \left.-\int_{0}^{t}\left(t_{1}-s\right)^{H-v_{2}} d B(s, \omega)\right\}
\end{aligned}
$$

It is obvious that FBM is a time-dependent process. However, the increments of FBM are strict sense stationary (SSS), known as fractional Gaussian noise (FGN). For convenience, $\omega$ will be omitted in this paper. When FBM is sampled and the sampled FBM will be called DFBM. Another counterpart, DFGN is obtained from the increment of DFBM. For convenience, they are defined as follows:

$$
B_{H}[n]=B_{H}\left(n T_{s}\right)
$$

denotes the process of DFBM, where $T_{s}$ is the sampling time and

$$
x_{H}[n]=B_{H}[n]-B_{H}[n-1]
$$

the process of DFGN. It can be shown that DFGN is a discrete-time, Gaussian distributed, stationary process with zero mean and its ACF, denoted as $r_{H}[k]$, can be evaluated as

$$
\begin{gathered}
r_{H}[k]=E\left\{x_{H}[n+k] x_{H}[n]\right\}= \\
\frac{\sigma^{2}}{2}\left(|k+1|^{2 H}-2|k|^{2 H}+|k-1|^{2 H}\right)
\end{gathered}
$$

where $\sigma^{2}$ is the variance of $x_{H}[n][6]$. The $\mathrm{ACF}$, $r_{H}[k]$, behaves asymptotically as $k^{2 H-2}$, which decays hyperbolically to zero other than exponentially. This property shows that DFGN possesses long-term correlation, which is easy to model certain complex processes by using one simple parameter. According to [6], [13], FGN possesses numerous important and useful properties. They can be rewritten as follows:

1)FGN has a zero mean.

2)FGN is a strict sense stationary process.

3) $\left.E\left\{B_{H}\left(t_{2}\right)-B_{H}\left(t_{1}\right)\right]^{2}\right\}=V_{H}\left(t_{2}-t_{1}\right)^{2 H}$

where

$V_{H}=\frac{1}{\Gamma(H+1 / 2)^{2}}\left\{\int_{-\infty}^{0}\left[(1-s)^{H-1 / 2}-(-s)^{H-1 / 2}\right]^{2} d s+\frac{1}{2 H}\right\}$

4) $\operatorname{Pr}\left\{\frac{B_{H}\left(t_{2}\right)-B_{H}}{\left(t_{2}-t_{1}\right)^{\prime \prime}} \frac{\left(t_{1}\right)}{2}<x\right\}=F(x)$

where $F(x)$ is a Gaussian cumulative distributed function with zero mean and variance one.

5) $\left\{B_{H}(t+\tau)-B_{H}(t)\right\} \equiv\left\{h^{-H}\left[B_{H}(t+h \tau)-B_{H}(t)\right]\right\}$

where - means that two random functions have the same finite joint distribution functions, i.e., $\left\{B_{H}(t+\tau)-B_{H}(t)\right\}$ is self-similar.

Besides, another definition that will be applied is referred to as the entropy. Generally, the entropy implies the Shannon entropy other than more generalized Rényi entropy [14]. The entropy $H(X)$ of discrete random variable $X$ is defined by [14]

$$
H(X)=-\sum_{x \in S} p(x) \log p(x)
$$

where $S$ denotes the support set of random variable and $p(x)$ the probability mass function. The differential entropy $h(X)$ of a continuous random variable 
$X$ with a density $f(x)$ is defined as [14]

$$
h(X)=-\int_{S} f(x) \log f(x) d x,
$$

where $S$ denotes the support set of random variable. The differential entropy will be applied in this paper, since DFGN is a jointed Gaussian distributed. The entropy possesses some properties:

(a) $h(X+c)=h(X)$,

This property implies that translation does not change the differential entropy.

$$
\begin{aligned}
\text { (b) } h(a X) & =h(X)+\log |a| \text { and } \\
h(A X) & =h(\mathbf{X})+\log |A|
\end{aligned}
$$

where $|A|$ is the absolute value of determinant.

This property implies that a scaling factor just shift one constant up or down but does not change the form of curve.

(c)If $X$ is a jointly Gaussian distributed, then the entropy is

$$
h(X)=\frac{1}{2} \log (2 \pi e)^{N}|R|,
$$

where $R$ denotes the correlation matrix.

Besides, since $R$ is a Hermitian and Toeplitz matrix, we can use Levinson's algorithm [15] to effectively calculate the determinant of $R$. This Levinson's algorithm is expressed as follows:

1 . The recursive algorithm is initialized by

$$
a_{1}[1]=\frac{r[1]}{r[0]}, p_{1}=\left(1-\left|a_{1}[1]\right|^{2}\right) r[0]
$$

2.When $k>1$, the algorithm recursively computed as

$$
\begin{aligned}
& a_{k}[k]=\frac{r[k]-\sum_{i=1}^{k-1} a_{k-1}[i] r[k-i]}{p_{k-1}} \\
& a_{k}[i]=a_{k-1}[i]-a_{k}[k] a_{k-1}^{-}[k-i], i=1,2, \ldots, k-1 \\
& p_{k}=\left(1-\left|a_{k}[k]\right|^{2}\right) p_{k-1} .
\end{aligned}
$$

3. The reflection coefficients are given by $k_{k}=a_{k}[k]$. 4. If $p_{k-1}-p_{k} \leq \varepsilon$ exits, otherwise go to Step 2, where $\hat{\varepsilon}$ is some threshold.

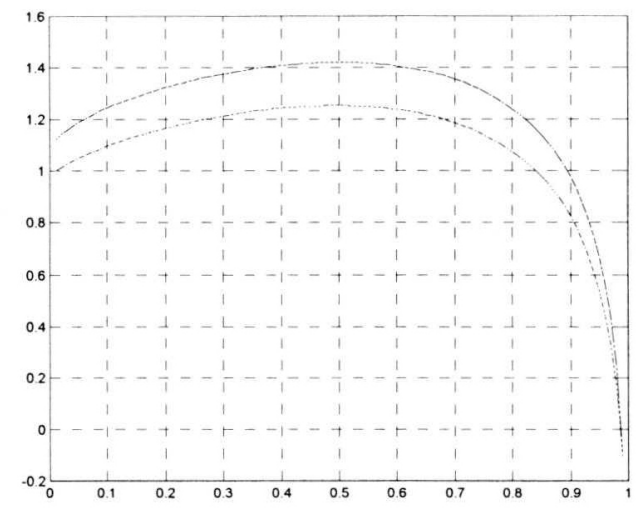

Fig. 1 The true and estimated average entropies for different $H=0.01: 0.01: 0.99$, where $N=100$, where the higher curve of this figure is the true average entropy and the other one is the estimated average entropy.

\subsection{The Entropy of DFGN}

It is well known [6] that the DFGN is a jointly Gaussian distributed random process,

$$
p(X ; H)=\frac{1}{(\sqrt{2 \pi})^{N}|R|^{1 / 2}} \exp \left\{-\frac{1}{2} X^{T} R^{-1} X\right\}
$$

where $X=[x[1], x[2], \ldots, x[N]]^{T}$ is the data set with zero mean and the correlation matrix

$$
R=E\left\{X X^{T}\right\}
$$

whose elements are $r_{H}[k]$ 's, as in (3). Therefore, according to (6), we have

$$
h(X)=\frac{1}{2} \log (2 \pi e)^{N}|R|,
$$

where $|R|$ is the determinant of autocorrelation matrix $R$. This determinant can be efficiently calculated according to the following equation:

$$
|R|=\prod_{k=0}^{N-1} p_{k}
$$

where $p_{k}$ 's are the prediction error power for the $k$ th order linear predictor in Levinson's algorithm. For convenience to application, we normalize the entropy by a factor of $N$, data length, as 


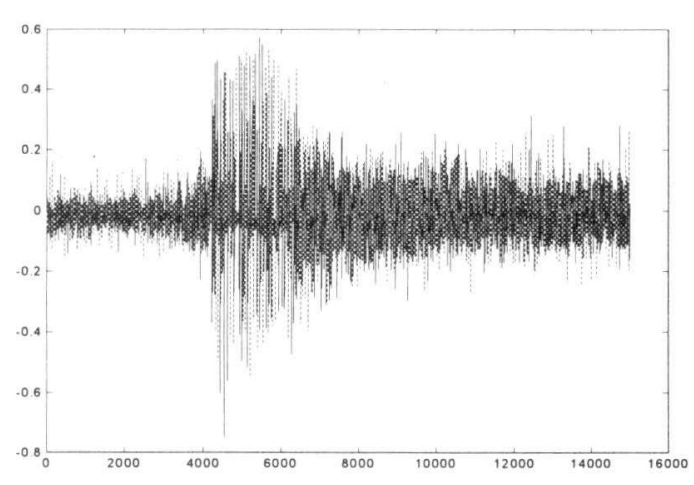

Fig. 2 The time series of the EUS EMG on the intact rat under room temperature of the bladder for one complete cycle of micturition.

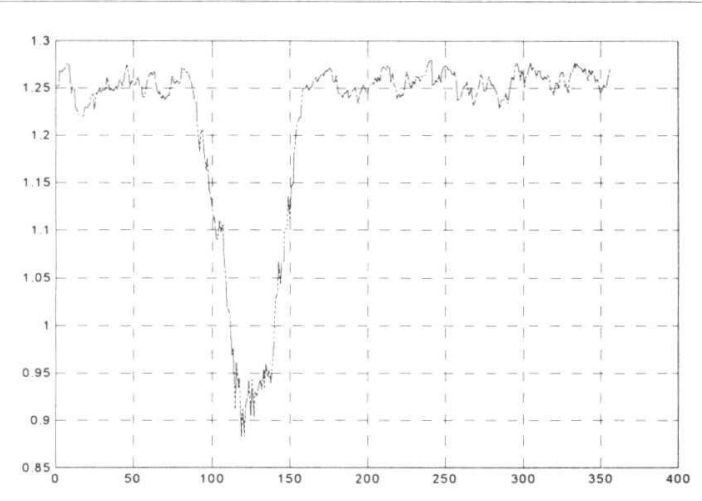

Fig. 3 The average entropies of the EUS EMG on the intact rat under room temperature of the bladder for one complete cycle of micturition.

$$
\frac{1}{N} h(X)=\frac{1}{2}\left(\log (2 \pi e)+\frac{1}{N}|R|\right),
$$

which will be referred to as average entropy (a.e.) It is obvious that the average entropy is almost the same, as $N$ is large enough. Fig. 1 illustrates the true and estimated average entropies for different $H$, where "true" and "estimated" imply that average entropies come from true and estimated ACF respectively.

This figure shows that the maximum of entropy happens at $H=0.5$. It is considerably reasonable, since the DFGN with $H=0.5$ is white Gaussian noise. Intuitively, we anticipate that the entropy is symmetric with $H=0.5$. However, this figure tells us it is asymmetric. For application, it can be implemented as a four-step algorithm.

\subsubsection{Algorithm}

1. Select adequate data record, $N$, for real signal.

2. Estimate the ACF of real data by using a biased es- timator, which is

$$
\hat{r}[k]=\frac{1}{N} \sum_{n=k+1}^{N} x[n] x[n-k]
$$

for $k=0,1, \ldots, N-1$.

3.Calculate the prediction error powers $p_{k}$ 's by using estimated ACF via Levinson's algorithm.

4. Calculate the determinant of autocorrelation matrix, $|R|$ and average entropy using (11) and (12).

\subsection{Applications}

The entropy is sometimes used for medical signal classification and identification. For instance [16], it facilitates the concept of symbolic dynamics, based on a coarse-graining of dynamics, to analyze the dynamic aspect of heart rate variability (HRV). These time series are first transformed into symbolic sequences with symbols from a given alphabet. Under this situation, some detailed information is lost in this procedure. However, the coarse dynamic behavior can be analyzed, since small changes of the process do not evidently influence the results. Next, a few of symbols are combined into a sequence of words. Then using Shannon or Rényi entropies to calculate their entropies from the distribution of woras. Higher values of these entropies posses higher complexity and lower values lower one. A low HRV is related to an increased risk for severe ventricular arrhythmia and sudden cardiac death (SCD). These phenomena are associated with a decreased complexity of beat-to-beat interval dynamics.

In this work, we use the proposed method on the external urethral sphincter electromyogram (EUS EMG) of the intact rats under both room temperature and cold-water stimulation of the bladder and the SCI rats. Their EUS EMGs and average entropies are illustrated by Fig. 2-7. For Fig. 2, Fig. 4, and Fig. 6, we use the time series of EUS EMG for one complete cycle of micturition. For Fig. 3, Fig. 5 and Fig. 7, we use the following procedure to compute.

\subsection{Procedure}

1. Select the first 800 points (i.e., data records $N=800$ ) to calculate its average entropy by using prior algorithm.

2. Shift time series to the right by 40 (i.e., 5 percent) points and sclect 800 points to calculate its average entropy.

3. Repeat Steps 1-2 until the number of remaining points is fewer than 800 points. 


\section{RESULTS}

Obviously, it is difficult to discriminate their differences from the time series of the EUS EMG as shown by Fig. 2, Fig. 4, and Fig. 6, respectively. However, remarkable differences derived from our proposed method are manifested. From Fig. 3, Fig. 5 and Fig. 7 we find that the average entropies can give us enough information to discriminate among themselves. First, we consider the intact rats under room temperature. In Fig. 3, the average entropies of the EUS EMG of the filling phase are fluctuating around 1.25 . The average entropies of the voiding phase or the bursting episode of EUS EMG can reach about 0.9 , and only one deep trough exists. The average entropies of the relaxing phase of EUS EMG again rise to around 1.25. Secondly, in Fig. 5, the intact rats under cold-water stimulation are considered. In this casc, the average entropies of the filling phase of the EUS EMG are also fluctuating around 1.25. The avcrage entropies of the voiding phase of EUS EMG, however, can only reach about 1.05 , and there exists many troughs. The average

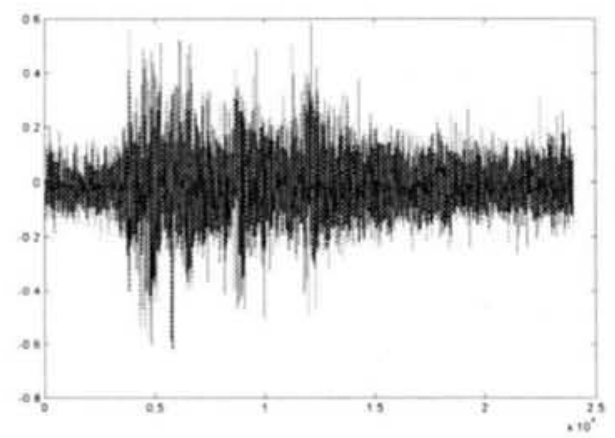

Fig. 4The time series of the EUS EMG on the intact rat under cold-water stimulation of the bladder for one complete cycle of micturition

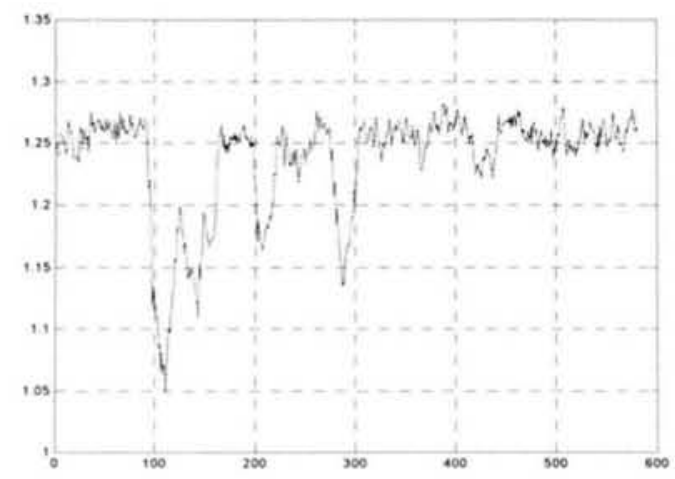

Fig. 5 The average entropies of the EUS EMG on the intact rat under cold-water stimulation of the bladder for one complete cycle of micturition. entropies of the relaxing phase of EUS EMG also rise to around 1.25. Finally, in Fig. 7, the SCI rats are investigated. In this case, no clear-cut valley exists, and the average entropies of EUS EMG are around 1.22 for three phases other than 1.25 .

\section{DISCUSSION}

From the time series, we cannot qualitatively discriminate the differences among three cases proposed, let alone quantitatively. In this paper, we propose an algorithm by using the entropy of DFGN to solve the problem. As we know that the entropy can be applied to calculate the measurc of uncertainty. The larger the entropy is, the more uncertain it will be. In the case of the rats under room temperature, the larger average entropies during the filling and relaxing phases can be inicrpreted as the muscle cells of EUS are not synergistic.

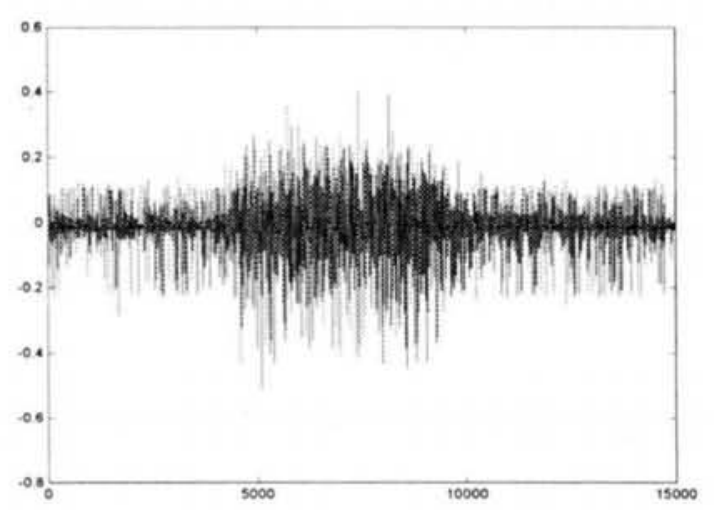

Fig. 6 The time series of the EUS EMG on the rat under SCI for one complete cycle of micturition.

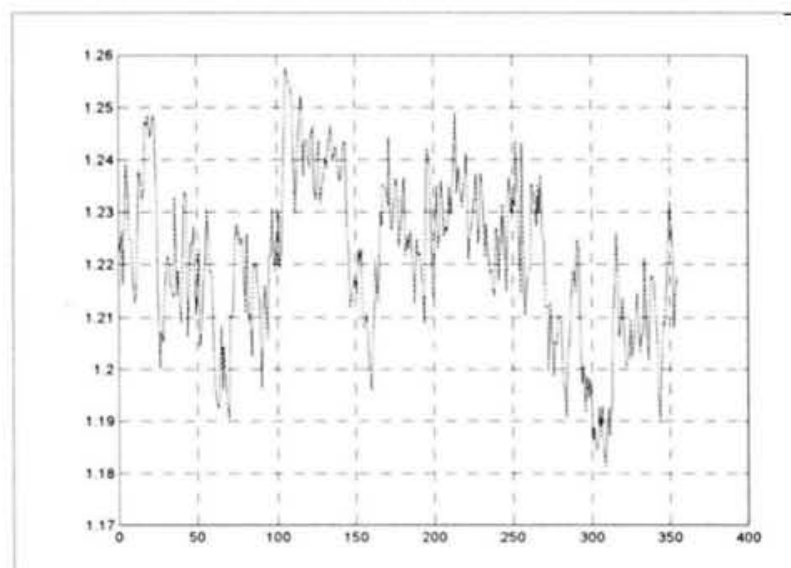

Fig. 7 The average entropies of the EUS EMG on the rat under SCI for one complete cycle of micturition. 
The lower average entropies during the voiding phase, however, can be interpreted as the muscle cells of EUS are more synergistic. Next, the situation of the rats under cold-water stimulation is similar to the one of the rats under room temperature during filling and relaxing phases. But many troughs during voiding phase can be interpreted as the muscle cells of EUS are less synergistic. As for the case of the $\mathrm{SCl}$ rats, the average entropies having no distinct trough means that the coordination mechanism is partly destroyed. From these results, we find that the synergia of the rats under room temperature is better than the one under cold-water stimulation, and much better than the one under $\mathrm{SCl}$.

\section{CONCLUSION}

We have derived the entropy of DFGN and found that this entropy is useful for analysis on the EMG of EUS signals. These analyses indicate that the muscle cells of EUS during the filling, relaxing and voiding phases can be quantitatively described according to our proposed algorithm. These results, when compared with our previous works [1] [2], they are consistent and offer a different perspective from information theory.

\section{REFERENCES}

1. Chang S, Mao ST, Kuo TP, Hu SJ, Lin WC, and Cheng CL: Fractal Geometry in Urodynamics of lower urinary tract. The Chinese Journal of Physiology 1999; 41: 25-31.

2. Chang S, Mao ST, Hu SJ, Lin WC, and Cheng CL: Studies of detrusor-sphincter synergia and dyssynergia during micturition in rats via fractional Brownian motion. IEEE 'Trans. Biomed. Eng. 2000; 47:1066-1073.

3. Falconer K: Fractal Geometry: Mathematical Foundations and Applications. John Wiley \& Sons, New York, USA, 1990; 246.

4. Liu SC and Chang S: Dimension estimation of discrete-time fractional Brownian motion with applications to image texture classification. IEEE Trans.
Image Processing 1997; 6: 1176-1184.

5. Chang YC and Chang $\mathrm{C}: \mathrm{A}$ fast estimation algorithm on the Hurst parameter of discrete-time fractional Brownian motion. IEEE Trans. Signal Processing (accepted as a regular paper)

6. Lundahl T, Ohley WJ, Kay SM, and Siffert R: Fractional Brownian motion: A maximum likelihood estimator and its application to image texture. IEEE Trans. Med. Imag. 1986; 5: 152-161.

7. Pentland AP: Fractal-based description of natural scenes. IEEE Trans. Pattern Anal. Machine Intell. 1984; 6: 661-674.

8. Kaplan LM and Kuo CCJ: Fractal estimation from noisy data via discrete fractional Gaussian noise (DFGN) and the Harr basis. IEEE Trans. Signal Processing 1993; 4: 3554-3562.

9. Sarkar $N$ and Chaudhuri BB: An efficient approach to estimation fractal dimension of textural images. Pattern Recognition Society 1992; 25: 1035-1041.

10. Sarkar $N$ and Chaudhuri BB: An efficient differential box-counting approach to compute fractal dimension of image. IEEE Trans. System, Man, and Cybernetics, 1994; 24: 115-120.

11.Jin XC, Ong SH, and Jayasooriah: A practical method for estimating fractal dimension," Patter Recognition Letters 1995; 16: 457-464.

12. Leu JS and Papamarcou A: On estimating the spectral exponent of fractional Brownian motion. IEEE Trans. Inform. Theory 1995; 41: 233-244.

13. Mandelbrot BB and Van Ness JW: Fractional Brownian motions, fractional noises and applications. SIAM Rev. 1968; 10: 422-437.

14. Cover TM and Thomas JA: Elements of Information Theory. John Wiley \& Sons, New York, USA, $1991 ; 499,13$, and 224.

15. Kay SM: Modern Spectral Estimation: Theory \& Application. Prentice-Hall , Englewood Cliffs, New Jersey, USA 1988: 170.

16. Voss A, Kurths J, Kleiner HJ, Witt A, Wessel N, Saparin P, Osterziel KJ, Schurath R, and Dietz R: The application of methods of non-linear dynamics for the improved and predictive recognition of patients threatened by sudden cardiac death. Cardiovascular Research 1996; 31: 419-433. 